\title{
EVALUATION OF RESOURCE OPTIMIZATION BASED ON QUANTUM SEARCH
}

\author{
SARA EL GAILY*1 \\ ${ }^{1}$ Department of Networked Systems and Services, Budapest University of Technology and Economics, \\ Múegyetem rkp. 3-9, Budapest, 1111, HUNGARY
}

\begin{abstract}
Quantum computing and communications try to identify more efficient solutions to the most challenging classical problems such as optimization, secure information transfer, etc. This paper will describe a new quantum method for the distribution of resources in computing platforms that consist of a large number of computing units. Furthermore, a simulation environment was developed and the performance of the new method compared to a classical reference strategy will be demonstrated. Moreover, it will be proven that the proposed solution tackles the problems of computational complexity, computing units that are time-consuming and slow to process, as well as the accuracy in determining the optimum result.
\end{abstract}

Keywords: quantum computing, quantum existence testing, finding extreme values in an unsorted database, resource management distribution, exhaustive algorithm, heuristic algorithm, computational complexity

\section{INTRODUCTION}

Nowadays, resource management and especially the distribution of resources is one of the most discussed and fundamental issues, for example, the problem considers a large amount of distributed energy resources including electric vehicles with gridable capacity [1], and the rapid progress of cloud computing, i.e. the growing number of video providers that have deployed their streaming services onto multiple distributed data centers [2]. This greatest demand on efficient resource distribution motivated us to search for new approaches and solutions.

The majority of operational processes require an amount of computational resources and their wide availability, namely the amount of computing units, is not the present issue, rather the utilization of resources and usage of the units are [3]. Thus, this challenge was tackled by introducing a new strategy which aims to optimize the computational load of the resources that handle the problems of computational complexity, time, speed and accuracy.

As is common knowledge, the primary aim of Quantum Computing and Communications is to reduce computational complexity and achieve optimum and efficient results with regard to the requirements of the given problem [4]. In order to exploit the power of quantum computing in terms of resource distribution, a quantum extreme value searching algorithm was used [5], which will be

\footnotetext{
*Correspondence: elgaily@hit.bme.hu
}

combined with an appropriately designed classical framework.

\section{Modelling}

Our novel model deals with the classical problem of resource distribution and contains three components.

The first one generates tasks to be computed in the system denoted by $p_{i}$ and can be characterized as processing time, memory, energy, etc. The actual number of running tasks in the system is referred to as $n$. These tasks are served by computing units that represent the resources of the system. The number of the units is denoted by $c$. The computing units may have different theoretical capacities. The theoretical capacity of the $j^{\text {th }}$ unit is depicted as $s_{j}$ and its free (unused) capacity is denoted by $x_{j}[t]$ which depends on time $t$, as depicted in Fig. 1 .

The third block is the decision-making unit which answers the question of how to deploy a new task among the process units in order to optimize the operation of the

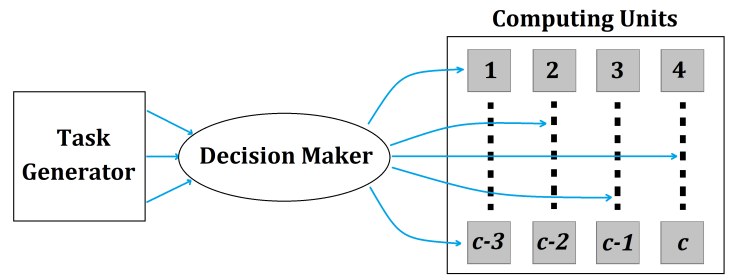

Figure 1: Architecture of the model 
system. There are different metrics to distribute the resources, here uniformly loaded units have been chosen because actually, they are an important aspect of many applications.

The overall capacity of the processing units in the system can be calculated as

$$
\widehat{s}=\sum_{j=1}^{c} s_{j},
$$

while the overall free processing capacity is determined according to

$$
\widehat{x}=\sum_{j=1}^{c} x_{j}[t] .
$$

The average amount of free capacity per unit is given by the following expression

$$
\bar{x}[t]=\frac{1}{c} \sum_{j=1}^{c} \frac{x_{j}[t]}{s_{j}} .
$$

Our purpose is to uniformly distribute the load over the resources. Therefore, the variance of the relative free capacities in the system is used. In the case of optimal task distribution, if $\sigma^{2}$ tends to zero, then the resources are distributed uniformly, otherwise they are not. The corresponding formula of the relative variance is:

$$
\sigma^{2}=\frac{1}{c} \sum_{j=1}^{c}\left(\bar{x}[t]-\frac{x_{j}[t]}{s_{j}}\right)^{2} .
$$

\subsection{Description of the quantum algorithm}

The optimized solution will be based on the quantum extreme value searching algorithm, a stochastic process which functions on an unsorted database that combines the technique of a classical binary search of a sorted database [6] and quantum existence testing (QET) [4]. The best classical solution requires $N$ queries of the database to determine the optimum result, while in order to solve previous problems the well-known logarithmic (often referred to as binary) search algorithm, which is originally intended to search for a given item in a sorted database with quantum existence testing (a special case of quantum existence testing interested in whether a given entry exists in the database or not rather than in determining the number of existence entries) needed to be combined. The quantum algorithm maintains the efficiency of the binary search while processing an unsorted database [4].

It is hard to classically compute the optimum deployment scenario, therefore, the quantum extreme value searching algorithm [5] is applied as a minimum searching algorithm (MSA), which enables the deployment scenario to be identified and results in minimum variance. The MSA is a stochastic process that works on an unsorted database. Our new approach handles the database
1: We start with $S=0$ :

$$
\begin{aligned}
& \sigma_{\min 1}^{2}=\sigma_{\min 0}^{2}, \\
& \sigma_{\max 1}^{2}=\sigma_{\max 0}^{2}, \\
& \text { and } \\
& \Delta \sigma^{2}=\sigma_{\max 0}^{2}-\sigma_{\min 0}^{2}
\end{aligned}
$$

2: $S=S+1$

3: $\sigma_{\operatorname{med} S}^{2}=\sigma_{\min S}^{2}+\left[\frac{\sigma_{\max S}^{2}-\sigma_{\min S}^{2}}{2}\right]$

4: $f l a g=\mathrm{QET}\left(\sigma_{\operatorname{med} S}^{2}\right)$

5: if $f l a g=$ True then

6: else $\sigma_{\max S+1}^{2}=\sigma_{\operatorname{med} S}^{2}$

$$
\begin{aligned}
& \sigma_{\max S+1}^{2}=\sigma_{\max S}^{2}, \\
& \sigma_{\min S+1}^{2}=\sigma_{\operatorname{med} S}^{2} \\
& \text { 7: if } S<\log _{2}\left(\frac{\Delta \sigma^{2}}{\alpha}\right) \text { then } \\
& \text { goto } 2 \\
& \text { 8: else } \begin{array}{c}
y_{\text {opt }}=\sigma_{\text {med } S}^{2} \\
\text { stop }
\end{array}
\end{aligned}
$$

as a function, i.e. the variance. The proposed algorithm is now given in detail:

The algorithm will stop once the following step $S<$ $\log _{2}\left(\frac{\Delta \sigma^{2}}{\alpha}\right)$ has been fulfilled where $\alpha$ denotes the smallest sub-region between two possible results in a database and is explained in more detail in Section 2.3.

\subsection{Description of the randomized, exhaus- tive and sequence-searching algorithms}

The randomized, exhaustive and sequential- searching algorithms are generally viewed as references and the cornerstone with regard to finding solutions. They are considered as three different methods [7]. Firstly, the randomized approach anticipates the solution guided by given knowledge and is not perceived as an optimal solution because it randomly searches for one solution in one step from the space in which the duration of time may be less reasonable [8]. On the other hand, the exhaustive algorithm examines every possible solution and leads to an optimal result, it checks all the $\mathcal{O}(d)$ steps which is time-consuming and yields an accurate result. A large number of iterations are required when compared to the number of queries, hence, its time-consuming nature. Furthermore, the sequence method exhibits a similar degree of computational complexity to the randomized method, using $\mathcal{O}$ (const) or $\mathcal{O}(1)$.

\subsection{Evaluation of the algorithms}

Our purpose is to provide a concrete comparison of solving distribution problems via heuristic 'random', exhaustive or sequence as well as quantum algorithms, hence, the difference in terms of the computational complexity applied and the minimum variance computed by each method was measured leading to the optimum distribution uniformity of the system. 


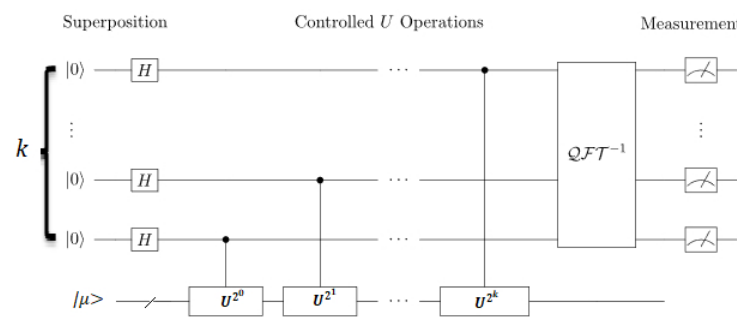

Figure 2: Quantum existing testing device (QFT: Quantum Fourier Transform).

\section{Comparison according to computational complexity}

By comparing the computational complexity of the quantum, randomized, exhaustive and sequence solutions, the quantum solution requires only $\sqrt{\log _{2}(T) \log _{2}^{3}(\sqrt{d})}$ steps, where $d$ refers to the number of different possible deployment scenarios and $T$ denotes the number of subregions given by all the possible outcomes of the different deployments. The exhaustive solution, on the other hand, needs $\mathcal{O}(d)$. With regard to the randomized solution, it uses fewer steps so is faster than the three former solutions, which means that the computational complexity of the random method is less than for the other ones. Moreover, it does not determine the optimum solution since the computational complexity of the sequence method is similar to the randomized algorithm. In contrast, the exhaustive and quantum solutions require more computation steps, but the quantum solution always is preferred because it requires significantly less computation than the exhaustive method.

As was mentioned previously, the quantum extreme value searching algorithm uses the binary search and quantum existing testing methods. As the quantum phase estimation algorithm is the core of QET, as can be seen in Fig. 2, it outperforms the other counterpart's algorithms, thus, its physical implementation is highly constrained with the required number of bits $k$.

In fact, the number of bits $k$ depends on the application of the system, as is illustrated in Fig. 2. This remains hard to realize, for example, if the error probability $P_{\varepsilon}$ of the application is neglected and the classical specification considered, which is of accuracy $a$, the number of steps needed is $\mathcal{O}\left(\log _{2}(T) \log _{2}^{3}(\sqrt{d})\right)$ and the number of bits will be influenced only by one factor, namely the accuracy $a$, as presented in

$$
k=a-1
$$

In addition, the relationship between the maximum relative variance and the number of sub-regions given $T$ according to all the possible outcomes of the different deployments is given by

$$
T=\frac{\sigma_{\max }^{2}}{\alpha}
$$

where $\alpha$ denotes the smallest sub-region between two possible results in a database (Eq. 7). $\alpha$ is illustrated in

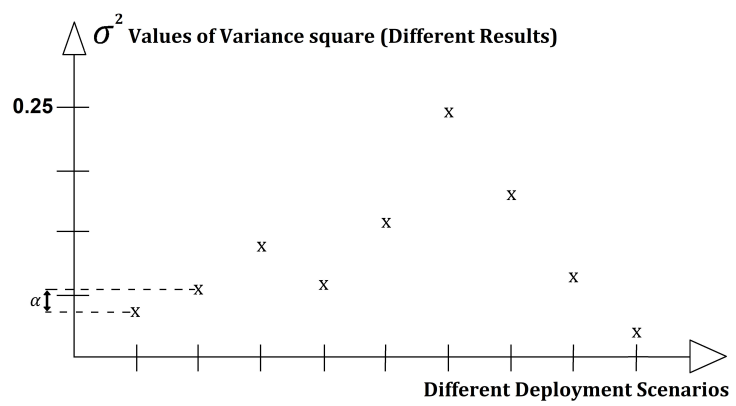

Figure 3: Functional representation of the database

Fig. 3:

$$
\alpha=\min _{\forall i, j}\left|\left(\sigma_{i}^{2}-\sigma_{j}^{2}\right)\right|
$$

On the other hand, if two factors, namely the accuracy $a$ and probability of error $P_{\varepsilon}$ [4], are taken into consideration, this assumption will influence the second term of the computational complexity $\log _{2}^{3}(\sqrt{d})$, it will be transformed into $\mathcal{O}\left(\log _{2}(T) \log _{2}^{3}\left(2^{\frac{P}{2}} \sqrt{d}\right)\right)$ and the number of bits will be expressed by

$$
k=a-1+\underbrace{\left[\log _{2}(2 \pi)+\log _{2}\left(3+\frac{1}{\check{P}_{\varepsilon}}\right)\right]}_{P},
$$

where $\check{P}_{\varepsilon}$ is the maximally allowed quantum uncertainty (probability of error) and $P$ is the number of qbit which controls the quantum uncertainty. The computational complexity of the classical (exhaustive) and quantum solutions is compared. The number of computational steps to yield the desired results with regard to the number of different deployment scenarios is in accordance with function $\mathcal{O}(d)$, while the quantum solution only requires $\mathcal{O}\left(\log _{2}(T) \log _{2}^{3}(\sqrt{d})\right)$.

Furthermore, the quantum method derives its stochastic behavior from the quantum phase estimation algorithm which heightens the degree of accuracy and speed in computation.

In the light of what has been shown, in Fig. 4, the classical strategy for identifying the deployment scenario which leads to the minimum variance needs more computational computing, while the quantum solution requires significantly less computation, which reduces the complexity and duration necessary to determine the optimum deployment scenario of resource distribution.

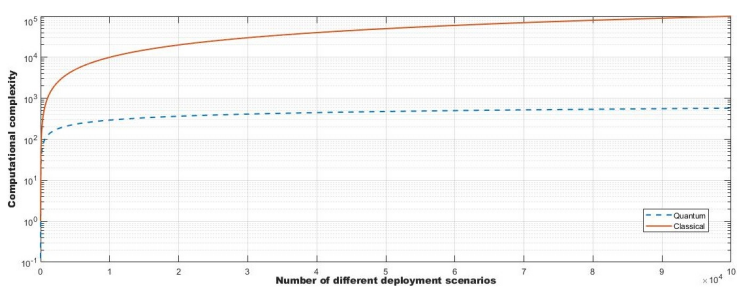

Figure 4: Comparison between the computational complexity of the classical and quantum decision-makers 


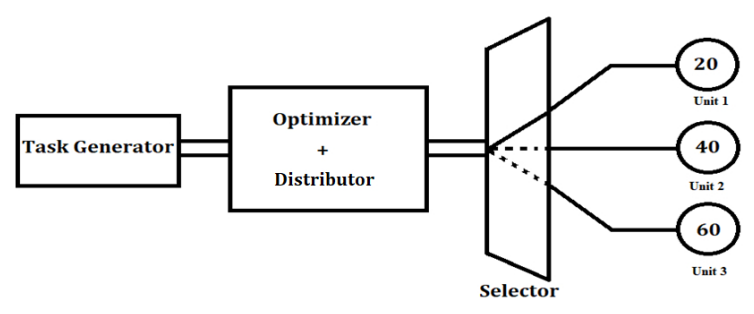

Figure 5: The simulation architecture

\section{Comparison of the uniformity}

In compliance with what has been discussed, the quantum and exhaustive solutions conserve the uniformity of the system, but the proposed quantum solution is the best and most efficient method because it requires less computation and time to determine the optimum deployment scenarios. However, the randomized and sequence algorithms do not ensure the uniformity of task distribution.

\section{Results and Analysis}

To show the importance of the proposed quantum solution, a simulation environment of Optimizer+Distributor was constructed.

The model of a resource distribution system contains three processing units with different theoretical capacities: twenty, forty and sixty running tasks in parallel. Practical systems contain significantly more processing units, however, to observe trends and effects it is worthwhile investigating a small-scale model first. Furthermore, a task generator block is present in the system. The tasks are considered to have exponential arrival times. An Optimizer+Distributor block is considered to be a decision-maker between the different deployment scenarios. The model implemented in the simulation environment is illustrated in Fig. 5.

\subsection{Experiments}

Two factors influence the behavior of the simulation: the mean (intensity) of the exponential distribution with re-

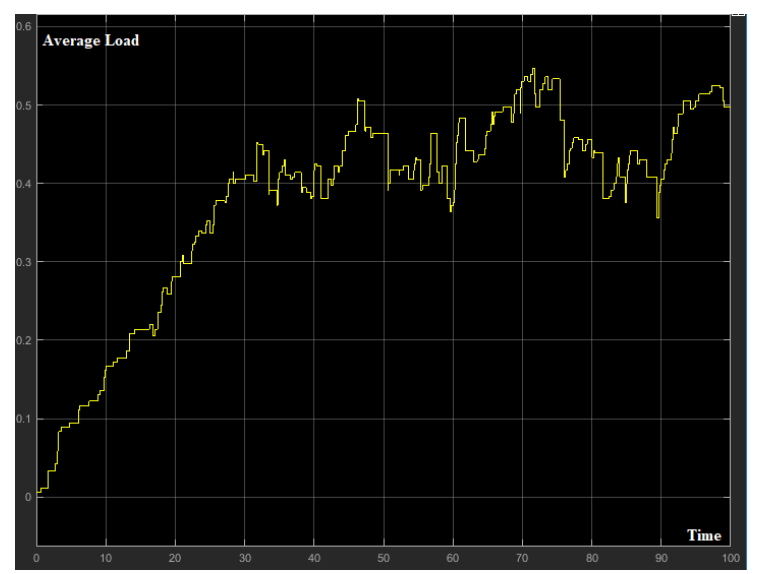

Figure 6: The average load of processing units in the case of the random reference strategy

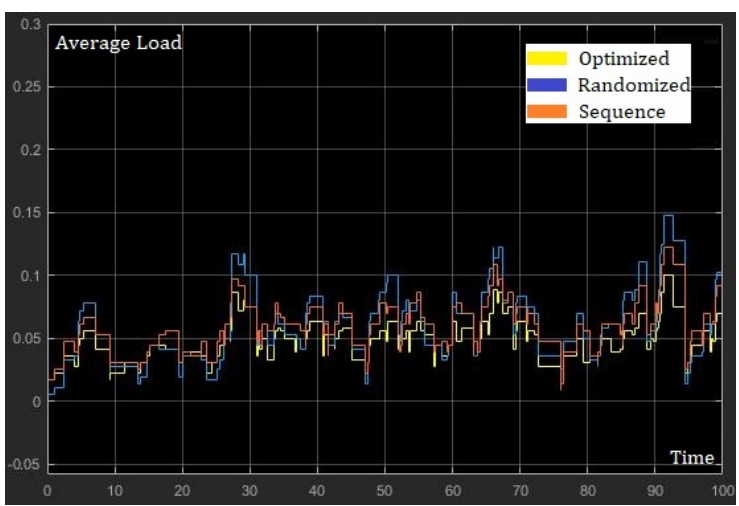

Figure 7: Average load of the three distribution strategies when the mean (intensity) of the exponential distribution of the arrival times of tasks is equal to 0.6

gard to the arrival times of tasks, and the service time of the tasks in the processing units.

The system will be more heavily loaded if the mean value is smaller or the service time of the tasks larger.

\subsection{Simulations}

In order to demonstrate the efficiency of the proposed optimization strategy, two other reference strategies were considered which distribute the tasks randomly or sequentially among the processing units.

By considering the following simulation parameters, the mean of the exponential arrival times is equal to 0.4 and the service time of the resources is equal to 3 .

The average load of the processing units in the case of the random reference strategy is presented in Fig. 6 with the following simulation parameters: the mean of the exponential arrival times is equal to 0.4 and the service time of the resources is equal to 15 . The line graph contains two phases: the transition phase with a duration of 0 to $30 \mathrm{~s}$, and the stationary phase that commences after approximately $30 \mathrm{~s}$, when the system reaches a certain equilibrium.

Comparing the performance of the three distribution strategies, it can be stated that during the transition phase the variances of the reference systems are approximately stable, but during the stationary phase (normal operation) the variance started to fluctuate dramatically as well as increase. On the other hand, the variance of the proposed quantum solution remained approximately linear and tended to zero, therefore, the quantum system conserves the distribution uniformity.

According to Fig. 7 and 8 the average load of the different methods remains similar independent of the intensity of the exponential distribution of the arrival times of tasks as well as the decision methods. Furthermore, it is clearly noticeable that when the mean of the exponential distribution of the arrival times of tasks is smaller, the tasks are generated faster which leads to an increase in the load of the computing units. 


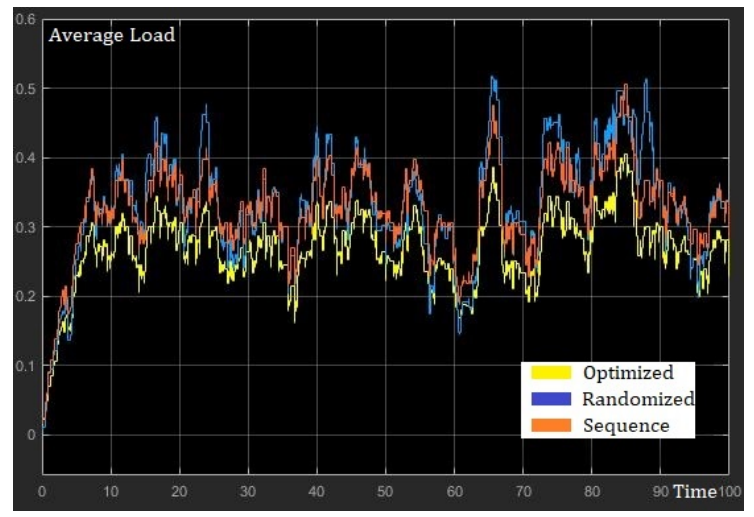

Figure 8: Average load of the three distribution strategies when the mean (intensity) of the exponential distribution of the arrival times of tasks is equal to 0.1

The simulation results concerning the variance of each distribution strategy show that the trends of the randomized and sequence strategies diverge from zero, but the sequence method yields a more uniform variance than the randomized one. In contrast, the optimized (quantum and exhaustive) strategies maintain and conserve the load uniformity of the system. These results are illustrated in Fig. 9 and 10.

\section{Conclusion}

In this paper, a new strategy for resource distribution based on a quantum searching algorithm was introduced. It was demonstrated that the quantum solution is more efficient by comparing the computational complexity and distribution uniformity of the quantum solution with the randomized, exhaustive and sequence methods. Furthermore, the changes applied to the computational complexity when the specification classical requirement depends on the application were demonstrated.

To show the importance of the quantum solution, a simulation environment of the proposed optimization of

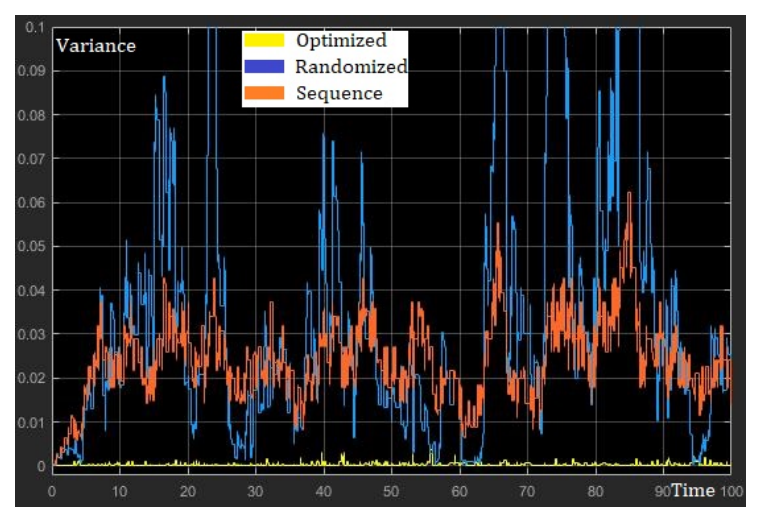

Figure 9: Variances over time of the optimized (yellow), randomized (blue) and sequence distribution (orange) strategies when the mean (intensity) of the exponential distribution of the arrival times of tasks is equal to 0.1

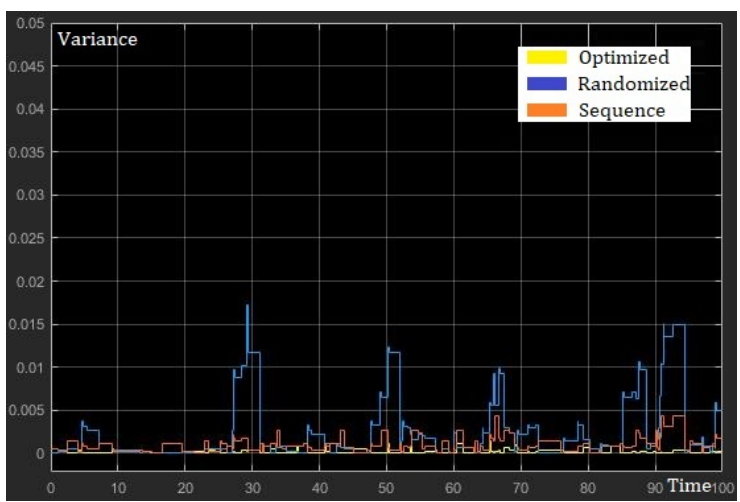

Figure 10: Variances over time of the optimized (yellow), randomized (blue) and sequence distribution (orange) strategies when the mean (intensity) of the exponential distribution of the arrival times of tasks is equal to 0.6

a distribution system was constructed and compared to two reference distribution systems which follow the randomized and sequence strategies. The proposed quantum approach is practical in most domains of infocommunication and computer science where resources have to be distributed among a large number of processing units.

These are the initial results of this new field of research. Obviously, other optimization metrics should be considered in the future. Furthermore, tasks can be modelled in a more sophisticated manner: different classes of tasks can be defined following various arrival processes and characterized by more than one resource parameter (processing time, memory, battery requirement). Finally, even an individual task can contain more blocks with dependencies among them and different blocks can be distributed among different processing units.

The wider future context of our research is to start with a polynomial time problem and progress towards a nondeterministic polynomial time problem.

\section{Acknowledgement}

The research was partially supported by the National Research Development and Innovation Office of Hungary (Project No.2017-1.2.1-NKP-2017-00001), by the Hungarian Scientific Research Fund -OTKA K-112125 and in part by BME Artificial Intelligence FIKP grant of EMMI (BME FIKP- MI/SC). The research reported in this paper was supported by the BME- Artificial Intelligence FIKP grant of EMMI (BME FIKP-MI/SC).

\section{REFERENCES}

[1] Soares, J.; Canizes, B.; Vale, Z; Venayagamoorthy, G. K.: Benders' decomposition applied to energy resource management in smart distribution networks, Clemson University Power Systems Conference, Clemson, SC. March 8, 2016. DOI: 10.1109/PSC.2016.7462820 
[2] Zhang, Z.; Jiang, X.; Xi, H.: Joint resource allocation and traffic management for cloud video distribution over software-defined networks, $8^{\text {th }}$ IEEE International Conference on Communication Software and Networks, University of Science and Technology of China, Hefei, China. 2016. DOI: 10.1109/ICCSN.2016.7586692

[3] Keränen, J.; Kortelainen, J.; Antila, M.: Computational resource management system. Version 1.0/14.8.2015. https://www.vtt.fi/inf/julkaisut/ muut/2015/VTT-R-01975-15.pdf

[4] Imre, S.; Balázs, F.: Quantum computing and communications: An engineering approach (Wiley, Chichester, UK) 2005 ISBN 0-470-86902-X, DOI: 10.1002/9780470869048

[5] Imre, S.: Extreme value searching in unsorted databases based on quantum computing, Int. J. Quantum Inf., 2005 3(1), 171-176 DOI: 10.1142/S0219749905000700

[6] Knuth, D. E.: Sorting and searching in The art of computer programming Vol. 3 ( $2^{\text {nd }}$ ed.) (AddisonWesley, Reading, MA, USA) 1998 ISBN 978-0-20189685-5

[7] Zin, N. A. M.; Abdullah, S. N. H. S; Zainal, N. F. A.; Ismail, E.: A comparison of exhaustive, heuristic and genetic algorithm for travelling salesman problem in PROLOG, Int. J. Adv. Sci. Eng. Inf. Technol., 2012 2(6), 459-463 DOI: 10.18517/ijaseit.2.6.244

[8] Hooker, J. N.: Toward unification of exact and heuristic optimization methods, Int. T. Oper. Res., 2013 22, 19-48 DOI: 10.1111/itor.12020 